\title{
The Influence of Consolidation on Shear Test Results
}

\author{
G. HAAKER \\ Faculty of Mechanical Engineering, University of Twente, P.O. Box 217, 7500AE Enschede (The Netherlands)
}

(Received January 6, 1986; in revised form February 1, 1987)

\section{SUMMARY}

Measurements of the yield behaviour of a cohesive bulk solid in the Jenike shear tester are normally done in two steps. First, pre-shearing to a steady state yielding, and secondly, shearing under a lower normal stress to establish the shear stress at incipient failure.

Due to the limited strain of the sample in this apparatus, the material must be consolidated in an earlier stage. This is done by the twisting action of a loaded cover, at which the vertical load and the amount of twists are chosen such that a suitable stress-strain curve will be obtained in the pre-shearing stage. The ratio $n$ between the vertical load during consolidation and pre-shearing, respectively, can have a strong impact on the results.

In this article, results of measurements with various $n$-values are given, leading to a variation in shear stress levels for both steady state and incipient failure. This can be explained by variations in the real normal stresses on the shear plane, which can substantially deviate from the externally applied normal stresses, due to contact between ring and base. To establish the real normal stress on the plane of shear, a somewhat modified shear tester was used. Furthermore, measurements were performed where the contact between ring and base was avoided by a removeable pair of half-rings.

The results of those measurements and the implications for the derived quantities are amply discussed, resulting in some recommendations for a more reliable testing procedure.

\section{INTRODUCTION}

The Jenike shear tester [1] is a popular device for measuring the failure properties of bulk solids.
In this apparatus, a sample is first presheared under a known vertical load until a steady state shearing condition is attained. From there on, the vertical load is decreased and shearing continued to establish the shear stress for incipient failure. The normal and shear stresses from both the steady state and incipient failure from series of tests enable the so-called yield loci, from which the relevant flow properties can be derived, to be constructed.

Unfortunately the apparatus allows only a small horizontal strain to reach the steady state, so the sample must be consolidated somehow in an earlier stage. This is done via the twisting action of a loaded cover, the vertical load and the amount of twists being chosen such that a suitable stress-strain curve will be obtained in the pre-shearing stage. The choice of the ratio $n$ between the vertical load during consolidation and the one during pre-shear, however, can strongly influence the shear stress levels from both steady state and incipient failure. This is mainly caused by variations of the real normal stresses on the plane of shear which can substantially deviate from the external applied normal stresses, due to the contact force between ring and base. It could be one of the reasons for the deviations in the results of measurements performed on the same material, but in different laboratories. In the following sections, the results and implications of research work are described in which this phenomenon is given thorough attention.

\section{PRELIMINARY MEASUREMENTS}

In earlier research work already published [2], we modified the base of the shear tester such that the real force $K$ on the plane of shear could be measured. This construction is given in Fig. 1. 


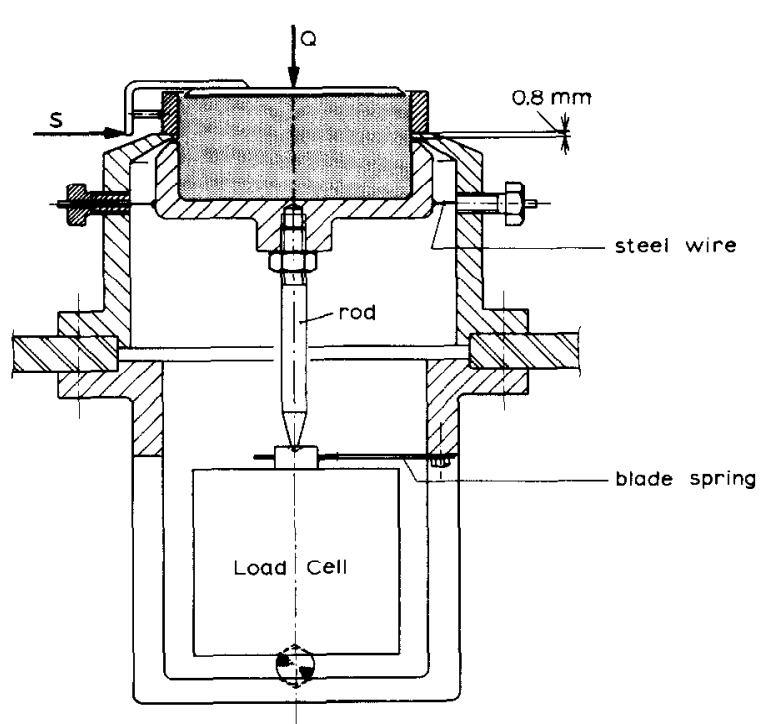

Fig. 1. Measurement of the real normal force $K$ on the shear plane.

Via a stiff rod the base is supported by a load cell which measures the vertical load on the base. The vertical position of the base can be adjusted by the length of the rod. Horizontal movement is constrained by four adjustable steel wires attached to the base, while a blade spring fixes the central position of the load cell. A rigid housing placed around the base ends in a thin ring $(\simeq 0,8 \mathrm{~mm})$ above, but free from the upper edge of the base, over which the upper ring can slide. This construction enables us to measure the value of $K$ directly, since no force can be transmitted by the ring to the base.

From previous measurements with this construction, it was found that the real normal force $K$ starts to rise on the run to incipient failure. This is due to the dilation of the material, which causes the ring-base contact force to decrease or even lifts the ring from the base. This phenomenon may explain the variation in stress level sometimes experienced when trying to reach a steady state shearing condition.

Let us consider for instance Fig. 2, where results of some measurements with a calcite powder are given. Curve a represents the stress-strain relation (horizontal shear force as a function of the horizontal displacement of the ring) of the pre-shearing stage for a sample that was consolidated during the twisting action with a load ratio $n=1$. Curve a can be considered to represent a well-

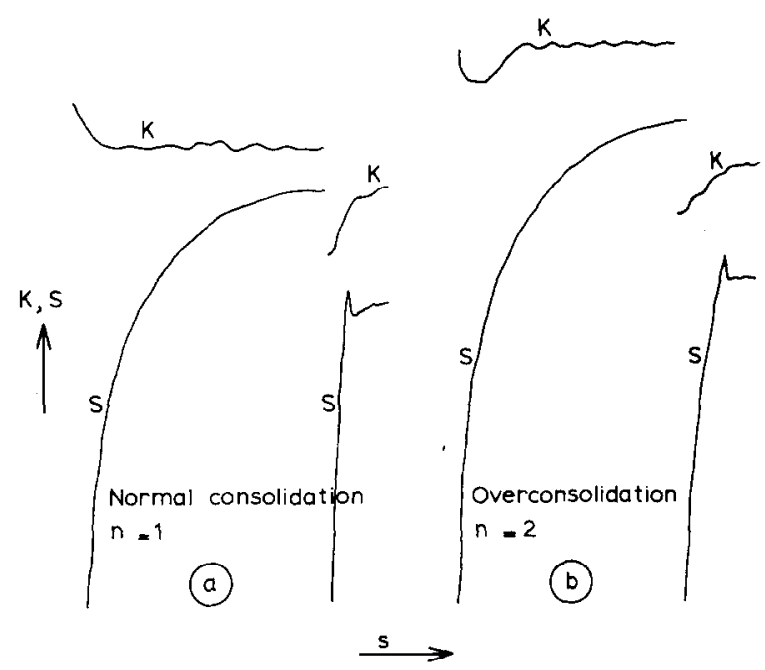

Fig. 2. Curves of stress-strain as a function of load ratio.

defined steady state shearing condition. Now if we increase the load ratio for instance to $n=2$, we can expect the sample to behave as an overconsolidated sample, which means that the stress-strain curve for pre-shearing should pass a maximum value before it levels off to the steady state.

However, if we look at curve $b$ which represents the case with $n=2$, we see again a well-defined steady state shearing condition, although at a higher level compared with curve a. Without further information, it is difficult to decide which curve should be taken as a base for further measurements, because with no doubt different yield loci and derived values will be obtained for both cases. An explanation for this behaviour can be found if we look at the real normal forces $K$ on the shear plane, also shown in the figure. From those curves we see that in case $b$ the value of $K$ is significantly higher, most probably because the initially overconsolidated sample starts to expand which causes the contact force between ring and base to decrease. Due to the increase of $K$, the sample no longer behaves as an overconsolidated sample an a good stress-strain steady state curve can be obtained, although at a higher level.

The implications of this phenomenon will be studied further in the next paragraphs, but it can already be concluded from these preliminary results that the consolidation level can have a significant influence on shear test results. This will certainly hamper the 
interpretation if no further information is available about the real forces on the shear plane, which is normally the case.

\section{MEASUREMENTS AND RESULTS WITH VARI- OUS LOAD RATIOS}

To establish to what extent the value of the load ratio $n$ could influence the results, we did series of measurements on a calcite powder with $n=1$ and $n$ as high as possible.

The highest acceptable value of $n$ is restricted by the fact that the sample is not allowed to be overconsolidated by the twisting action. To determine the maximum $n$ value, series of tests were performed with stepwise increase of $n$ until the stress-strain curve of the pre-shearing stage tended to pass a maximum value before leveling off. The acceptable maximum $n$-values were a little bit depending on the actual normal stress levels, but for the three yield loci considered they were in the range of $n=2.41-2.17$. In all our tests we twisted twenty times, one twist being a rotation over an angle of $90^{\circ}$ and vice versa. During twisting, the filling ring was allowed to rotate, and rotation of the shearing ring was prevented by hand.

The results of tests with $n=1$ for three yield loci are given in Fig. 3 in the usual manner, that is, the measured shear stresses against the external applied normal stresses (solid lines representing a straight-line approximation of the yield loci).

The results of tests with $n=2.17-2.41$ are also reported in Fig. 3 (dashed lines being a straight-line approximation of the yield loci). It is seen immediately from this figure that the higher $n$-values lead to a significantly higher position of the yield loci. This is not surprising if we consider the values of the real normal force $K$ on the shear plane in both situations. Those values of $K$ can best be compared if we express them as a percentage of the external applied load $N$ (including the weight of the lid, the weight of the upper ring and the weight of the bulk material above the plane of shear).

For $n=1$ we measured $K / N$ ratios ranging from $0.68-0.73$ for the steady state and from $0.76-0.85$ for the shearing points of the three yield loci. In the case of $n=2.17$ -

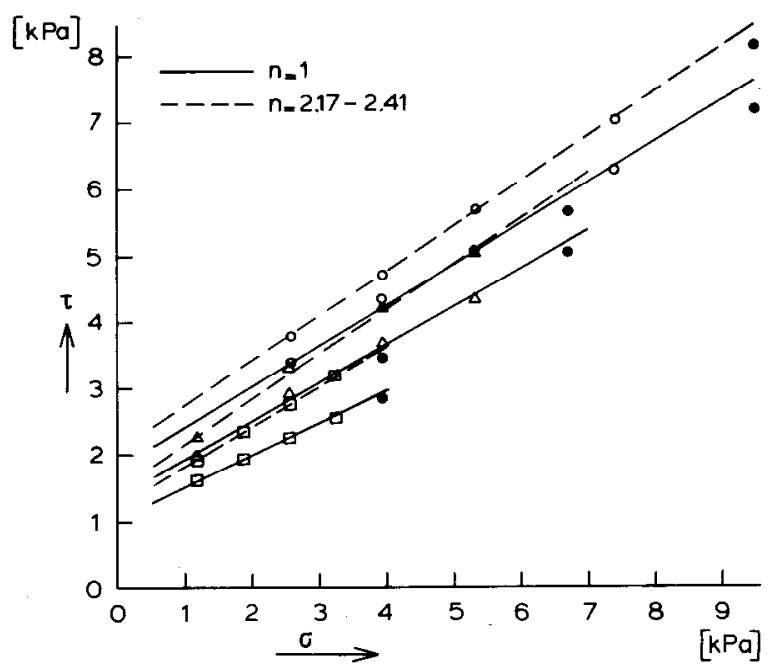

Fig. 3. Results of shear tests with $n=1$ and $n=2.17$ 2.41 .

2.41, however, those values were much higher, ranging from 0.90 to 0.93 for the steady state and from 0.9 to 1 for the shearing points.

From the figure we can conclude that the influence of the high $n$-values on the result is in fact twofold. First, during twisting and preshear, the sample is consolidated under a high normal load (measured as a higher $K$ value in the tests), leading to a more consolidated material. Secondly, during the shearing stage, this higher consolidated sample is brought to shear again under a higher real normal load (or K-value) compared with the case of $n=1$. For these two reasons, the differences between the two pairs of yield loci are not surprising. In Fig. 4, the results derived from the yield loci are given, i.e., the unconfined yield strength $\sigma_{p}$ and the effective internal friction angle $\phi_{e}$ as a function of the major consolidating stress from the steady state $\sigma_{1}$. Again it is seen that the results with $n=2.17-2.41$ are remarkably higher than those with $n=1$.

At a first glance, one might think that a better approach would be to take the value of $K$ instead of the external applied vertical load in order to calculate the normal stresses for the points of the yield loci. We have not done this, however, for two reasons. First, in the normally constructed shear tester, the values of $K$ can not be measured and we do not want to deviate too far from the normal situation. Secondly, if one takes $K$-values, which are in fact corrected values for the 


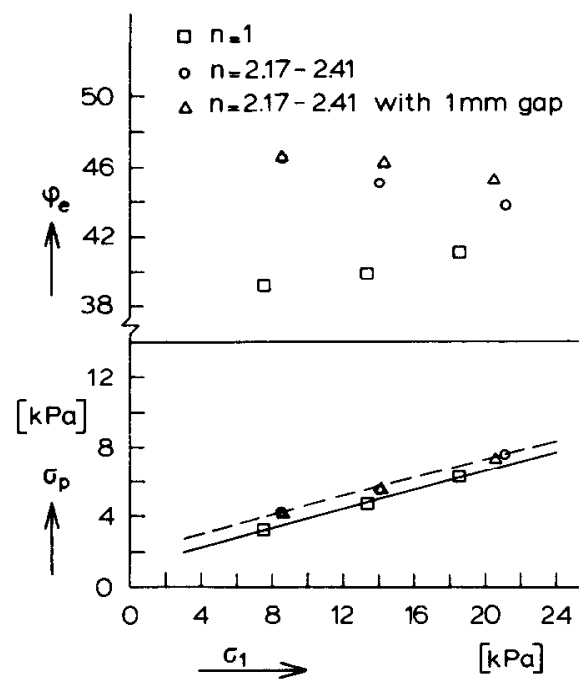

Fig. 4. Results derived from yield loci of Fig. 3 and Fig. 5.

external applied normal stress, one would also have to correct the shear stress for the contact between ring and base. Instead of this, we tried to create a situation where the values of $K$ are close to the externally applied load, as is the case in the measurements with the high $n$ ratios. In the next section a different approach is described.

\section{MEASUREMENTS WITH NO CONTACT BETWEEN RING AND BASE}

In earlier measurements described elsewhere [2], we tried to lift the ring by hand from the base half-way through the preshearing stage. This however, was not a complete success, probably due to the fact that it is virtually impossible to lift the ring purely horizontally. So here we practised a more reliable method.

To avoid contact between ring and base during the relevant stages of the test, we placed a pair of half-rings $1 \mathrm{~mm}$ thick between ring and base. After loading and twisting, these two half-rings were removed, and hence a gap of about $1 \mathrm{~mm}$ between ring and base was created at the start of preshearing.

With this device, we measured again at $n=$ 1 and $n=2.17-2.41$. However, with $n=1$, it proved impossible to reach a steady state within a reasonable strain. This is due to the fact that the normal stress on the plane

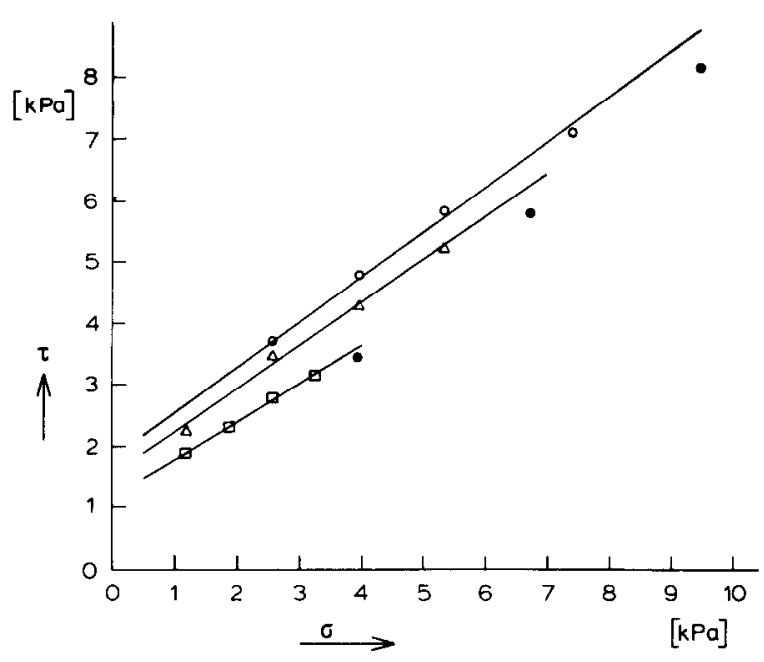

Fig. 5. Results from tests with the ring free from the base, $n=2.17-2.41$.

of shear during consolidation (twisting action) is lower than during pre-shearing, because in the latter case, the contact force between ring and base is eliminated. So the sample behaves as an underconsolidated sample in the pre-shearing stage.

For this reason, we only performed series of tests with $n=2.17-2.41$ in the same stress range as previous tests. For the lowest yield locus, we found $K / N$-ratios ranging from 0.94 to 0.99 , so a part, although very slight, of the external load is still not transferred to the plane of shear.

For the other two yield loci, we found in all tests $K / N \simeq 1$, which means that the externally applied load equals the vertical load on the plane of shear, the situation we wanted to obtain.

The results of these tests are given as yield loci (straight-line approximation) in Fig. 5. For comparison, the derived results are given in Fig. 4.

It is seen from these figures that the results at $n=2.17-2.41$ for both the normal tests and the free ring tests are very close, which could be expected because the $K$-values also deviated only slightly.

\section{DISCUSSION}

From the measurements with $n=1$, it is seen that the real normal force $K$ on the plane of shear deviates up to $30 \%$ from the external applied vertical load. One might 


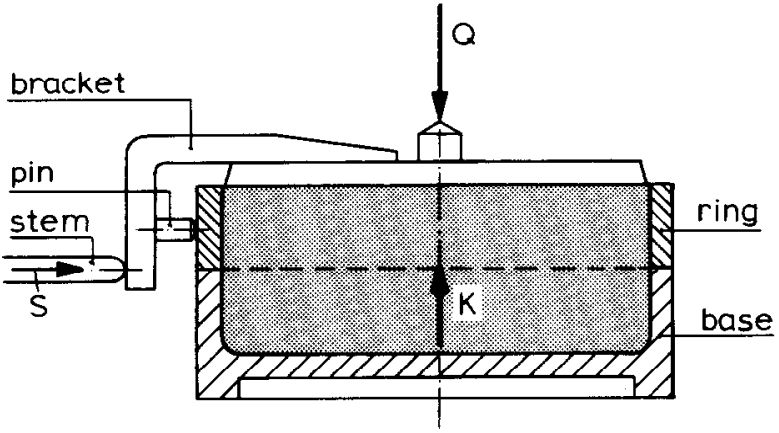

Fig. 6. Relevant forces for the ring-base contact force.

wonder how these large deviations can occur. The reasons for this are amply discussed in the literature [2], but some background is given here. The relevant situation is given in Fig. 6, where a contact is assumed between ring and base. Some of the forces work around the whole circumference of the ring. For reasons of simplicity, however, they are not drawn here in their exact positions.

Now, in a situation where the sample is consolidated with $n=1$, we can assume that the material still consolidates in the shearing stage before it reaches steady state. This means that the density of the material increases and the cover tends to move down a little. The contact force $F_{c}$ in this situation originates from three sources:

- The weight of the ring $W_{w}$.

- The silo effect in the upper ring, which causes a part of the vertical force $Q$ on the cover to be transmitted by friction to the wall, $W_{f}$. This effect can be amplified by the horizontal force introduced by the front side of the ring to the material when the sample is forced to shear. This influence is small, however, because at the rear of the ring, the horizontal force can decrease.

- As the cover moves down, or even tends to, the pin in the bracket will slide down the ring. The vertical movement of the latter is not possible because it is resting on the base. Due to the rather high contact force between pin and base $(\simeq S / 2)$, a considerable vertical friction force can be exerted on the ring, $W_{p}$.

During the shearing stage, the situation is reversed. Now the material starts to dilate and the friction force on the ring due to the material, as well as to the pin, will be reversed and the contact force between ring

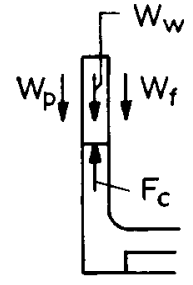

and base will decrease. The same will occur with the higher $n$-values, where the sample can behave as an overconsolidated sample in the pre-shearing stage. It is even possible that the real force $K$ in the plane of shear is higher than the externally applied load. This can be the case when the stem is stiff and a vertical friction force can be introduced between stem and bracket. For this reason, we did all our measurements with a long stem that could move freely.

It is obvious that all those problems are resolved when there is no contact between ring and base. In that case, a part of the load can only be transferred to the base by the material itself, but as long as the position of the ring is not too eccentric, this is negligible.

\section{CONCLUSIONS}

From the investigations described in this work, it can be concluded that the results of shear tests can be significantly influenced by the load ratio used for consolidation. If the load ratio chosen is too low, which is quite possible when starting with $n=1$, the real normal load on the plane of shear can be much lower than the externally applied load. This will lead to a significant underestimation of the flow function and the effective angle of internal friction. There are two possible ways to eliminate this problem.

First, instead of choosing the lowest possible load ratio $n$, which is normally done, the highest acceptable load ratio should be taken, taking care, however, not to overconsolidate the sample. In this manner, the sample starts to expand during pre-shearing and the contact 
force between ring and base decreases, so the actual load on the shear plane comes closer to the externally applied load. Second$\mathrm{ly}$, one can eliminate the contact force between ring and base by, for instance, a pair of thin half-rings between ring and base, which are removed before pre-shearing. In this manner, the total externally applied load is almost completely transferred to the material in the base. Due to the higher normal load on the plane of shear during preshearing, in this case too the load ratio chosen should not be too low, so that a steady state can be reached within a reasonable strain.

Both methods will lead to more reliable results, but on the basis of our measurements, the second method is to be preferred to the first.

\section{LIST OF SYMBOLS}

$F_{\mathrm{c}} \quad$ contact force between ring and base, N

$K$ real normal force on plane of shear, $\mathrm{N}$

$N$ externally applied load, including weight of cover, ring and material inside ring, $\mathrm{N}$ $n \quad$ load ratio, i.e., the ratio of externally applied load during consolidation and pre-shearing, -

$Q$ total vertical force on cover, $\mathrm{N}$

$S$ total externally applied horizontal force, $\mathrm{N}$

$W_{\mathbf{f}} \quad$ vertical friction force between ring and material inside, due to silo effect, $\mathrm{N}$

$W_{\mathrm{p}} \quad$ vertical friction force between pin and ring, $\mathrm{N}$

$W_{\mathrm{w}} \quad$ vertical force due to weight of ring, $\mathrm{N}$

$\sigma$ normal stress, $\mathrm{kPa}$

$\sigma_{1}$ major consolidating stress during preshearing, $\mathrm{kPa}$

$\sigma_{\mathfrak{p}} \quad$ unconfined yield strength, $\mathrm{kPa}$

$\tau \quad$ shear stress, $\mathrm{kPa}$

$\phi_{\mathbf{e}} \quad$ effective angle of internal friction, -

\section{REFERENCES}

1 A. W. Jenike, Storage and Flow of Bulk Solids, Utah Engng. Exp. Station, Univ, of Utah, Bulletin 123,1964 , revised 1970 .

2 F. J. C. Rademacher and G. Haaker, VDI-Forschungsheft, No. 629, June 1985, VDI-Verlag, Düsseldorf. 